\title{
Simvastatin induces growth inhibition and apoptosis in HepG2 and Huh7 hepatocellular carcinoma cells via upregulation of Notch1 expression
}

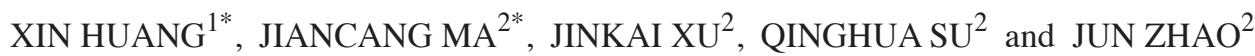 \\ ${ }^{1}$ Department of General Surgery, Xi'an Central Hospital, Xi'an Jiaotong University, Xi'an, Shaanxi 710003; \\ ${ }^{2}$ Department of General Surgery, The Second Affiliated Hospital of Xi'an Jiaotong University, \\ Xi'an, Shaanxi 710004, P.R. China
}

Received January 8, 2014; Accepted October 27, 2014

DOI: $10.3892 / \mathrm{mmr} .2014 .2976$

\begin{abstract}
Statins, cholesterol-lowering drugs, are one of the most commonly prescribed types of medications. Previous studies have suggested that simvastatin may inhibit the cell function and tumor growth of hepatocellular carcinoma (HCC) cells; however, the molecular mechanisms underlying simvastatin-induced apoptosis in HCC cells remains to be elucidated. The aim of the present study was to investigate the role of simvastatin in the regulation of cell viability, proliferation and apoptosis in HepG2 and Huh7 HCC cells, and to elucidate the specific regulatory mechanisms by which simvastatin proceeds. MTT, trypan blue and flow cytometric analyses were performed in order to detect viability, proliferation and apoptosis in HepG2 and Huh7 cells. The results of the present study demonstrated that simvastatin significantly decreased cell viability and proliferation as well as increased apoptosis in HepG2 and Huh7 cells compared to that in untreated cells. In addition, reverse transcription quantitative polymerase chain reaction and western blot analysis revealed that simvastatin-treated cells exhibited increased expression levles of Notch1, p53, and Bax, as well as decreased expression levels of B cell lymphoma 2; furthermore, Notch1 upregulation resulted in the inhibition of Akt phosphorylation. In conclusion, the results of the present study indicated that simvastatin significantly promoted apoptosis in $\mathrm{HCC}$ cells, the mechanism of which may have proceeded via the upregualtion of the Notch1 gene in the Akt-dependent signaling pathway.
\end{abstract}

Correspondence to: Dr Jun Zhao, Department of General Surgery, The Second Affiliated Hospital, Xi'an Jiaotong University, 157 Xi Wu Road, Xi'an, Shaanxi 710004, P.R. China

E-mail: zhaojunmed@163.com

*Contributed equally

Key words: hepatocellular carcinoma, simvastatin, apoptosis, Notch1, phosphorylated-Akt

\section{Introduction}

Hepatocellular carcinoma (HCC) accounts for $80-90 \%$ of primary liver cancers and is one of the most prevalent malignant tumors in the world (1). The incidence of HCC has steadily increased in Western countries over the past decade and the global incidence of $\mathrm{HCC}$ is predicted to continue to rise over the next few years $(2,3)$. HCC has the third-highest rate of cancer-associated mortalities (4) and therefore studies into the effective treatment of HCC are essential.

Statins, cholesterol-lowering drugs, are one of the most commonly prescribed types of medications. Previous studies have focused on the use of statins as therapeutic agents for the treatment of solid and hematological cancers $(5,6,7)$. Statins have been shown to elicit pleiotropic effects on various cell types and differentially modulate cellular functions, including cell migration, proliferation, survival and apoptosis, in normal as well as malignant cells (8). Simvastatin, a member of the statin family, was previously reported to regulate the migration, proliferation, apoptosis and growth of tumor cells $(9,10)$; in addition, Wang et al (11) demonstrated that simvastatin induced caspase-dependent apoptosis and activated p53 in OCM-1 cells. Kochuparambil et al (10) found that the inhibitory effect of simvastatin on prostate cancer cell growth occurred via the inhibition of the Akt pathway. Previous studies have suggested that simvastatin regulated arteriogenesis following stroke and enhanced the differentiation of bone marrow stromal cells into endothelial cells via the Notch signaling pathway $(12,13)$; this therefore indicated that the pharmacological manipulation of Notch signaling may be a promising novel strategy for the treatment of human disease.

Notch genes encode proteins which are activated via interaction with certain families of ligands $(14,15)$; in mammals, there are four Notch receptors (Notch1-4), which have five corresponding ligands, including $\delta$-like 1,3 and 4 as well as Jagged 1 and 2 . Interaction between Notch receptors and their ligands induces a two-step proteolysis, resulting in activation of the Notch receptors. Activated Notch receptors have a critical role in maintaining the balance between cell proliferation, differentiation and apoptosis (16); therefore perturbed 
Notch signaling may contribute to tumorigenesis. Notch1 is the primary member of the Notch family, which has been previously reported to regulate the growth, apoptosis, migration and invasion of tumor cells, including breast, pancreatic and cervical cancer cells (17-19). Qi et al 20) demonstrated that Notch1 regulated HCC cell proliferation and apoptosis via different mechanisms. Therefore, the aim of the present study was to investigate the effect of simvastatin on Akt signaling and, in turn, HCC cell proliferation and apoptosis, as well as to determine whether the mechanism of action of simvastatin proceeded via regulation of Notch1 expression.

\section{Materials and methods}

Cell culture. Human HCC HepG2 and Huh7 cells were obtained from the American Type Culture Collection (Manassas, VA, USA) and maintained in RPMI-1640 (Gibco-BRL, Carlsbad, CA, USA), as previously described (21), supplemented with $10 \%$ fetal bovine serum (FBS; HyClone Laboratories, Logan, UT, USA).

Simvastatin treatment. Simvastatin (Merck, Darmstadt, Germany) was dissolved in dimethyl sulfoxide (DMSO; Sigma-Aldrich, St. Louis, MO, USA) to obtain simvastatin concentrations of $0,2,4,8$ and $16 \mu \mathrm{M}$. HCC cells were plated and incubated with various concentrations of simvastatin $(50 \mu \mathrm{l})$ for 24,48 , and $72 \mathrm{~h}$ at $37^{\circ} \mathrm{C}$ in a humidified atmosphere of $5 \% \mathrm{CO}_{2}$ and $95 \%$ air. Following treatment, cells were collected and washed with phosphate-buffered saline (PBS; Sigma-Aldrich) prior to further use.

Trypan blue assay. A trypan blue exclusion assay was used to determine the viability of HepG2 and Huh7 cells. In brief, HepG2 and Huh7 cells were stained using $0.04 \%(\mathrm{w} / \mathrm{v})$ trypan blue solution (Invitrogen Life Technologies, Carlsbad, CA, USA) at room temperature for $7 \mathrm{~min}$, during which irreversibly damaged cell membranes take up the anionic dye trypan blue; cells which excluded trypan were considered to have survived and trypan blue-positive cells were identified as dead. HepG2 and Huh7 cells were then visualized using microscopy (magnification, x40). For each experiment, 200 HepG2 and Huh7 cells were analyzed from ten different fields of vision/dish.

MTT assay. HepG2 and Huh7 cells were seeded into 96-well plates at 1,000 cells per well and then treated with various concentrations of simvastatin. On the day cells were harvested, $100 \mu 1$ spent culture medium was replaced with an equal volume of fresh medium. MTT (Sigma-Aldrich) was added to the cells at a final concentration of $0.5 \mathrm{mg} / \mathrm{ml}$, and the plates were incubated for $4 \mathrm{~h}$ at $37^{\circ} \mathrm{C}$. DMSO $(100 \mu \mathrm{l})$ was then added to each well and plates incubated with agitation at room temperature for $10 \mathrm{~min}$. The absorbance was measured at $570 \mathrm{~nm}$ using a spectrophotometer (Multiskan MK3; Thermo Fisher Scientific, Waltham, MA, USA).

Notch1 small interfering (si)RNA plasmid transfection into HepG2 cells. Transfection of the Notch1 siRNA plasmid into HepG2 and Huh7 cells was performed as previously described (19). In brief, cells were plated in a 35-mm dish for
$24 \mathrm{~h}$ prior to transfection in complete medium. Transfection was performed using Lipofectamine 2000 (Invitrogen Life Technologies) according to the manufacturer's instructions. The Notch1-specific siRNA plasmid for HepG2 (sense, 5'-AAGGUGUCUUCCAGAUCCUGA-3') (22) and the scrambled sequence (5'-AAAUGUGUGUACGUCUCC UCC-3') were inserted into the pGPU6/green fluorescent protein/Neo plasmid (Shanghai GenePharma, Ltd, Shanghai, China). The efficiency of transfection was confirmed using western blot analysis. Clones, in which the expression of Notch1 was effectively suppressed, were selected and used for the in vitro study.

Detection of apoptosis. Cell apoptosis was analyzed using flow cytometric (FCM) analysis. In brief, following $48 \mathrm{~h}$ of simvastatin treatment at various concentrations $(0,2,4,8$ and $16 \mu \mathrm{M})$, HepG2 and Huh7 cells were trypsinized using trypsin (Mingzhu Chemical Co., Shanghai, China), washed with PBS and then suspended with binding buffer. Cells apoptosis was detected by Annexin V-fluorescein isothiocyanate (FITC)/propidium iodide (PI) kit (BD PharMingen, San Diego, CA, USA). Cells were incubated with $5 \mu \mathrm{l}$ PI (Sigma-Aldrich) for $15 \mathrm{~min}$ at room temperature in the dark, prior to suspension in $500 \mu \mathrm{l}$ binding buffer. The cells were then analyzed using FCM (Beckman-Coulter, Brea, CA, USA) for relative quantitative apoptosis.

Reverse transcription quantitative polymerase chain reaction $(R T-q P C R)$. Total RNA was isolated from transfected and non-transfected HepG2 cells using TRIzol ${ }^{\circledR}$ reagent (Life Technologies, Inc., Rockville, MD, USA) according to the manufacturer's instructions. Reverse transcription was performed on $1 \mu \mathrm{g}$ of total RNA from each sample using oligo (dT) primers and 200 units of SuperScript II (Life Technologies, Inc.) for extension. PCR amplification was performed using 1.25 U Ex Taq polymerase (Takara Bio, Inc., Dalian, China). All PCR products were resolved on $1.8 \%$ agarose gels containing ethidium bromide (Sigma-Aldrich). Primer pairs for human Notch1, p53, B cell lymphoma 2 (Bcl-2), Bcl-2-associated X protein (Bax), phosphorylated Akt (p-Akt), Akt and $\beta$-actin specific amplification of complementary DNA were as follows: Notch1 forward, 5'-CCGCAGTTGTGCTCCTGAA-3' and reverse, 5'-ACCTTG GCGGTCTCGTAGCT-3'; p53 forward, 5'-GACCCAGGT CCAGATGAAGCT-3' and reverse, 5'-ACCGTAGCTGCC CTGGTAGGT-3'; Bcl-2 forward, 5'-AGTTCGCCGAGATGT CCAGGCA-3' and reverse, 5'-ACTTGTGGCCCAGATAGG CACC-3'; Bax forward, 5'-ACAGGGTTTCACCAGGATC-3' and reverse, 5'-GCTGCCACCCGCAAGAAGAC-3'; p-Akt forward, 5'-GGAGAUCAUGCAGCAUCGC-3' and reverse, 5'-GCGAUGCUGCAUGAUCUCC-3'; Akt forward, 5'-CTT TCCAGACCCACGACC-3' and reverse, 5'-CTCCGAGTGCAG GTAGTCC-3'; $\beta$-actin forward, 5'-GAGGCACTCTTCCAG CCTTC-3' and reverse, 5'-GGATGTCCACGTCACACTTC-3'.

Western blot analysis. Cells were homogenized and lysed using radioimmunoprecipitation assay lysis buffer (100 mm NaCl; 50 mm Tris-HCl, pH 7.5; 1\% TritonX-100; $1 \mathrm{~mm}$ EDTA; $10 \mathrm{~mm} \beta$-glycerophosphate; $2 \mathrm{~mm}$ sodium vanadate; and protease inhibitor). Protein concentrations were assayed using the micro-bicinchoninic acid assay protein 
A

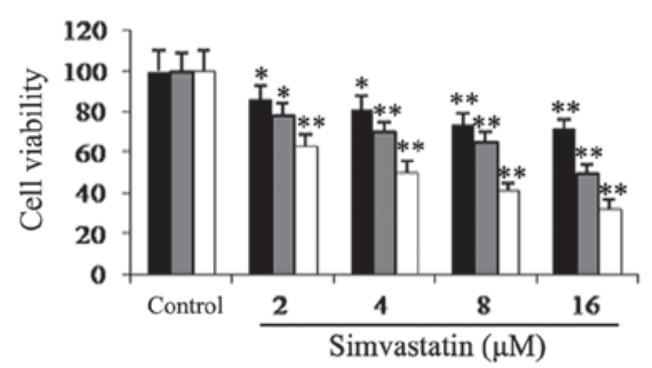

C

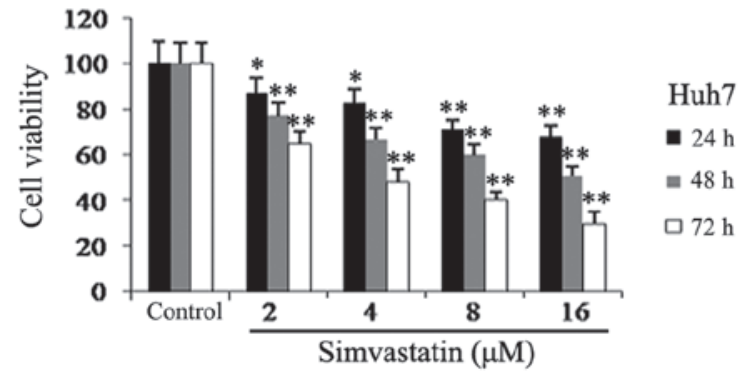

B

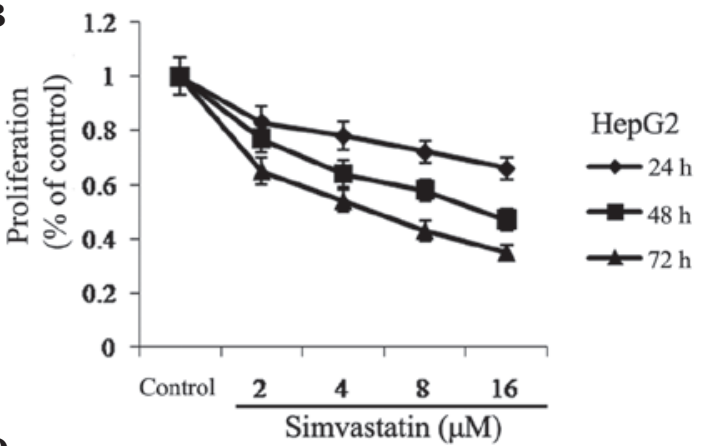

D

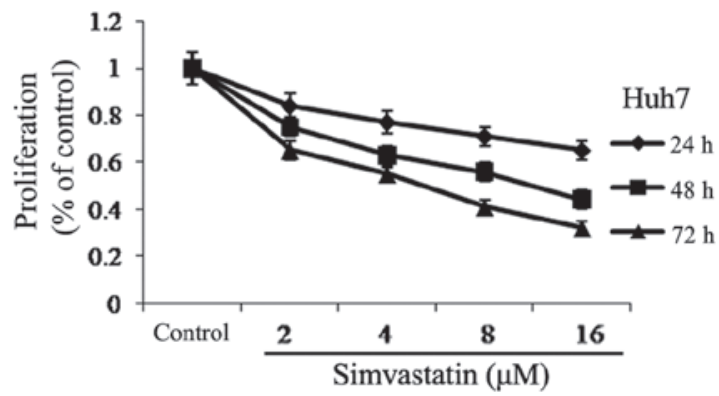

Figure 1. Simvastatin decreases HepG2 and Huh7 cell viability and proliferation. HepG2 and Huh7 cells were treated with various concentration of simvastatin $(0,2,4,8$ and $16 \mu \mathrm{M})$ for 24, 48 and $72 \mathrm{~h}$. (A) Bar graph showing trypan blue-positive simvastatin-treated HepG2 cells. (B) Broken line showing MTT results of proliferation of simvastatin-treated HepG2 cells. (C) Bar graph showing trypan blue-positive simvastatin-treated Huh7 cells. (D) Broken line showing MTT results of proliferation of simvastatin-treated Huh7 cells. ${ }^{*} \mathrm{P}<0.05$ and ${ }^{* *} \mathrm{P}<0.01$ compared with the control group.

assay (Pierce Biotechnology, Inc., Rockford, IL, USA). Proteins (20-30 $\mu$ g per lane) were separated using 10\% SDS-PAGE and then electroblotted onto nitrocellulose membranes (GE Healthcare, Little Chalfont, UK). Non-specific binding was blocked by incubating with $5 \%$ non-fat milk in PBS with Tween 20 buffer (Sigma-Aldrich) at room temperature for $1 \mathrm{~h}$. Cells were then incubated overnight at $4^{\circ} \mathrm{C}$ with a $1: 1,000$ dilution of primary antibodies for Notch1 (monoclonal mouse anti-human), Akt (polyclonal rabbit anti-human), p-Akt (polyclonal rabbit anti-human), p53 (polyclonal rabbit anti-human), $\beta$-actin (monoclonal mouse anti-human), Bcl-2 (monoclonal mouse anti-human) and Bax (monoclonal mouse anti-human), all purchase from Sigma-Aldrich, followed by incubation with the corresponding horseradish peroxidase-conjugated goat anti-rabbit immunoglobulin ( $\mathrm{Ig}) \mathrm{G}$ and rabbit anti-mouse IgG (1:2,000; Sigma-Aldrich) at room temperature for $1 \mathrm{~h}$. Antigens were then detected using the standard enhanced chemiluminescence kit (Santa Cruz Biotechnology, Inc., Dallas, TX, USA).

Statistical analysis. All experiments were performed in triplicate, unless otherwise stated. Statistical analyses were performed using SPSS 13.0 software (SPSS, Inc., Chicago, IL, USA). Values are presented as the mean \pm standard deviation. Statistical comparisons between groups were performed using a one-way analysis of variance followed by a Student's t test. $\mathrm{P}<0.05$ was considered to indicate a statistically significant difference between values.

\section{Results}

Simvastatin inhibits HCC cell viability and proliferation. The cytotoxic effects of various concentrations $(0,2,4,8$, and
$16 \mu \mathrm{M})$ and incubation periods $(24,48$, and $72 \mathrm{~h})$ of simvastatin was examined in HepG2 and Huh7 cells using a trypan blue assay. As shown in Fig. 1, simvastatin demonstrated high levels of cytotoxicity in HepG2 and Huh7 cells in a time- and dose-dependent manner. Following $72 \mathrm{~h}$ of simavastin treatment, even at the lowest dose $(2 \mu \mathrm{M})$, the viability and proliferation of HepG 2 and Huh7 cells was significantly decreased by $>34$ and $36 \%$, respectively, compared to the untreated group. These results therefore indicated that simvastatin may be a promising anti-cancer drug for the treatment of HCC.

Simvastatin induces apoptosis in HepG2 and Huh7 cells. $\mathrm{Bcl}-2$ family genes have an important role in the regulation of apoptosis; $\mathrm{Bcl}-2$ is an anti-apoptosis gene, while $\mathrm{Bax}$ is a pro-apoptosis gene (23). In normal cells the expression of $\mathrm{Bcl}-2$ and Bax is maintained at an equilibrium; however, during apoptosis, the expression of Bcl-2 is downregulated, while Bax is overexpressed (24). In the present study FCM analysis was performed in order to determine whether apoptosis contributed to the simvastatin-induced reduction in HepG2 and Huh7 cell viability. As shown in Fig. 2A and B, HepG2 and Huh7 cells in the experimental groups demonstrated a marked increase in apoptosis in a time- and dose-dependent manner. In addition, Fig. 2C and D showed that the apoptosis-associated genes Bcl-2 and Bax had significantly altered expression in cells treated with simvastatin; mRNA expression levels of Bcl-2 were significantly decreased and expression levels of Bax were significantly increased in a time- and dose-dependent manner.

Simvastatin increases mRNA expression levels of Notch1 and $p 53$. RT-qPCR was used in order to investigate the 
A

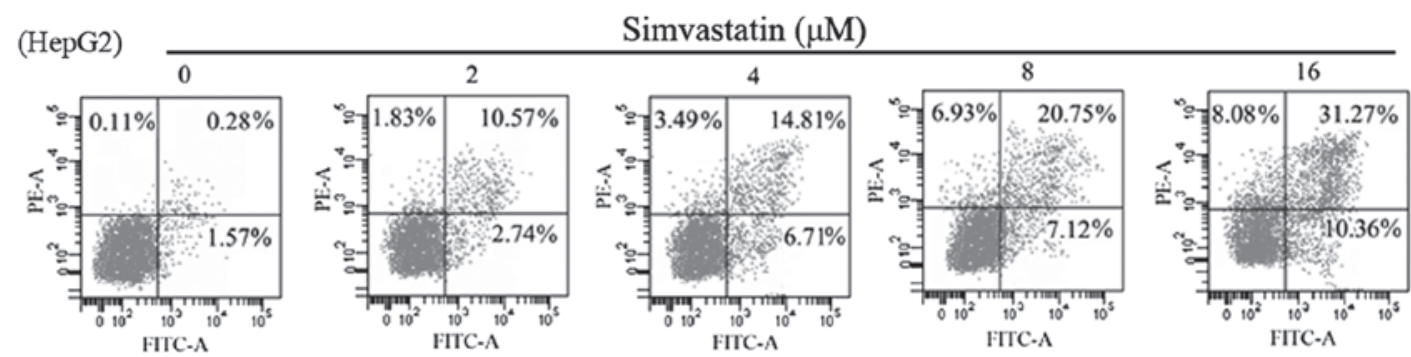

B

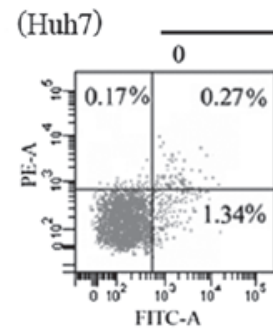

C

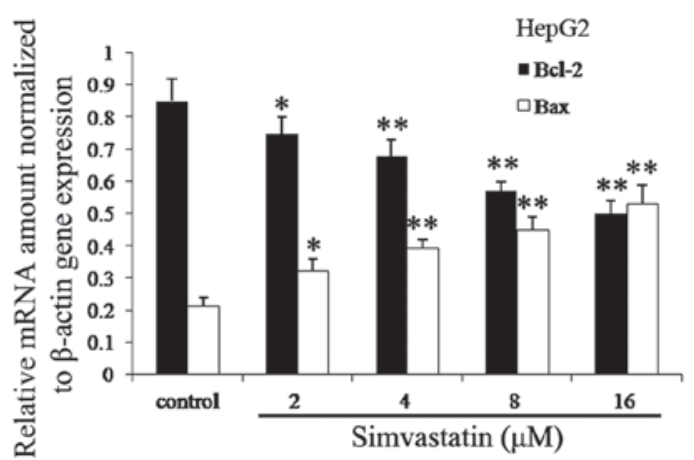

Simvastatin $(\mu \mathrm{M})$
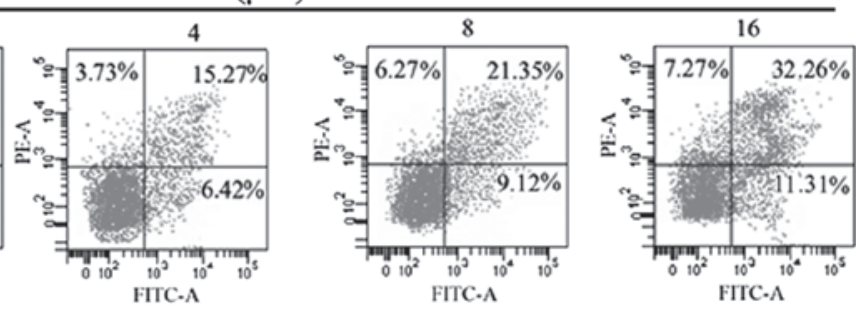

D

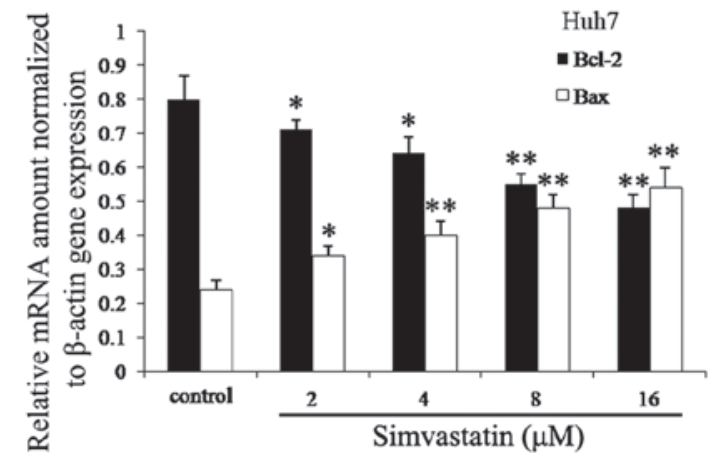

Figure 2. Simvastatin induces apoptosis and affects the expression of apoptosis-related genes in HepG2 and Huh7 cells. HepG2 and Huh7 cells were treated with various concentrations of simvastatin $(0,2,4,8$ and $16 \mu \mathrm{M})$ for $48 \mathrm{~h}$. Flow cytometric analysis of the apoptotic rate of simvastatin-treated (A) HepG2 and (B) Huh7 cells. (C) Reverse transcription quantitative polymerase chain reaction showing the mRNA expression level of Bcl-2 and Bax in simvastatin-treated (C) HepG2 and (D) Huh7 cells. ${ }^{*} \mathrm{P}<0.05$ and ${ }^{* *} \mathrm{P}<0.01$ compared with the control group. FITC-A, fluorescein isothiocyanate; PE, R-phycoerythrin; Bcl-2, B cell lymphoma 2; Bax, Bcl-2-associated X protein.

A

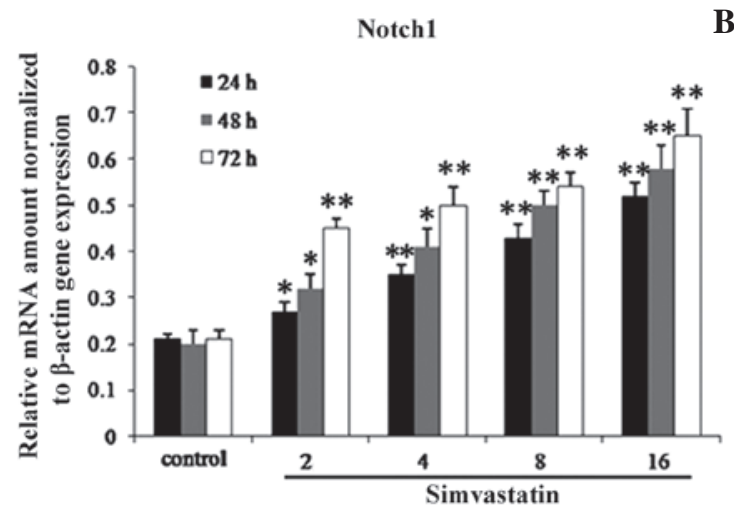

B

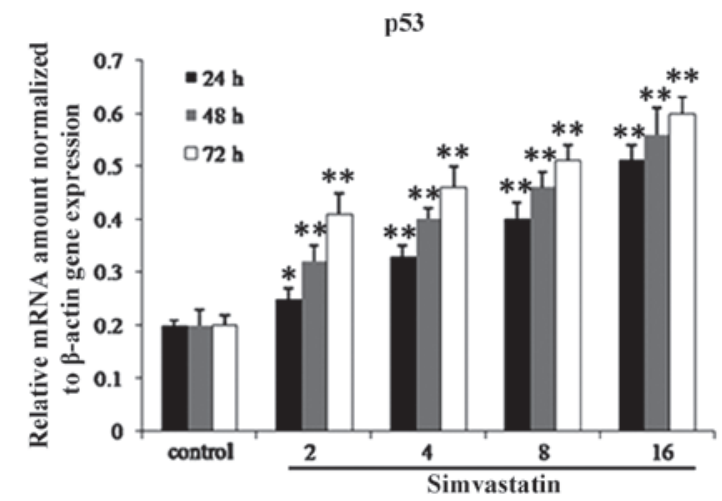

Figure 3. Simvastatin increases mRNA levels of Notch1 and p53 in HepG2 cells. Reverse transcription quantitative polymerase chain reaction showing the mRNA expression levels of (A) Notch1 and (B) p53 in HepG2 cells treated with various concentrations of simvastatin (0,2, 4, 8 and $16 \mu \mathrm{M})$ for 24,48 and $72 \mathrm{~h}$. ${ }^{*} \mathrm{P}<0.05$ and ${ }^{* *} \mathrm{P}<0.01$ compared with control group.

effects of simvastatin on the expression of Notch1 and p53 in HepG2 cells. As shown in Fig. 3, following incubation of HepG2 cells with various concentrations $(0,2,4,8$ and $16 \mu \mathrm{M}$ ) of simvastatin for $48 \mathrm{~h}$, mRNA expression levels of Notch1 and p53 were significantly increased in a dose-dependent manner.
Simvastatin-induced apoptosis is decreased following Notchl gene knock-out. The results of the present study, as shown above, demonstrated that simvastatin increased mRNA expression of Notch1; therefore, Notch1 siRNA transfection experiments were performed which knocked out the Notch1 gene in HepG2 cells, in order to further 
A

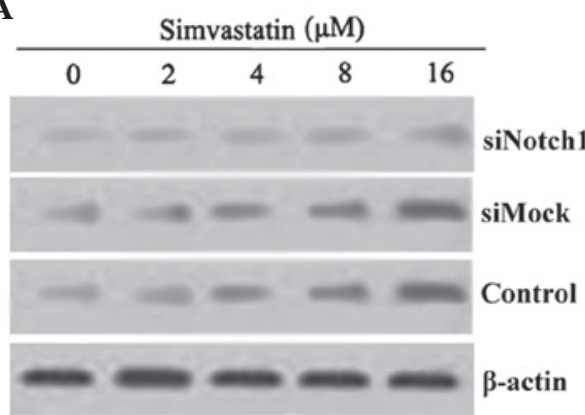

B

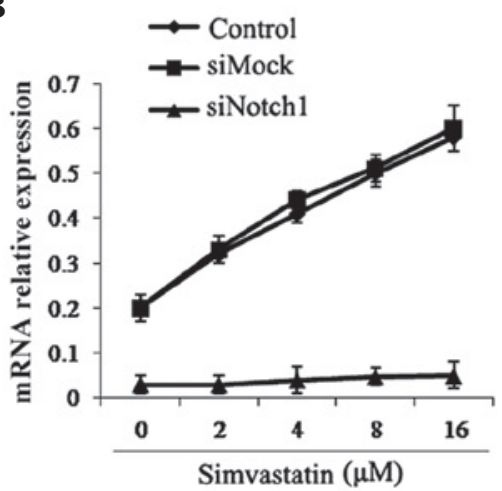

C

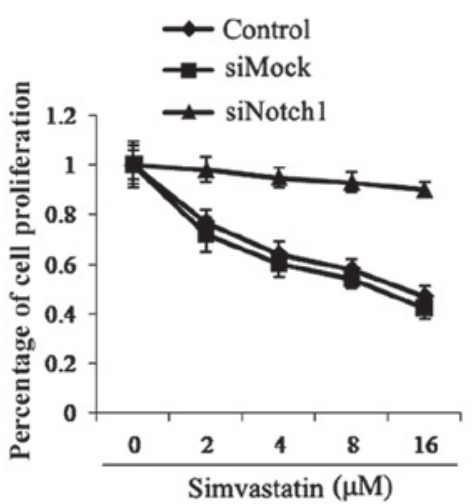

D

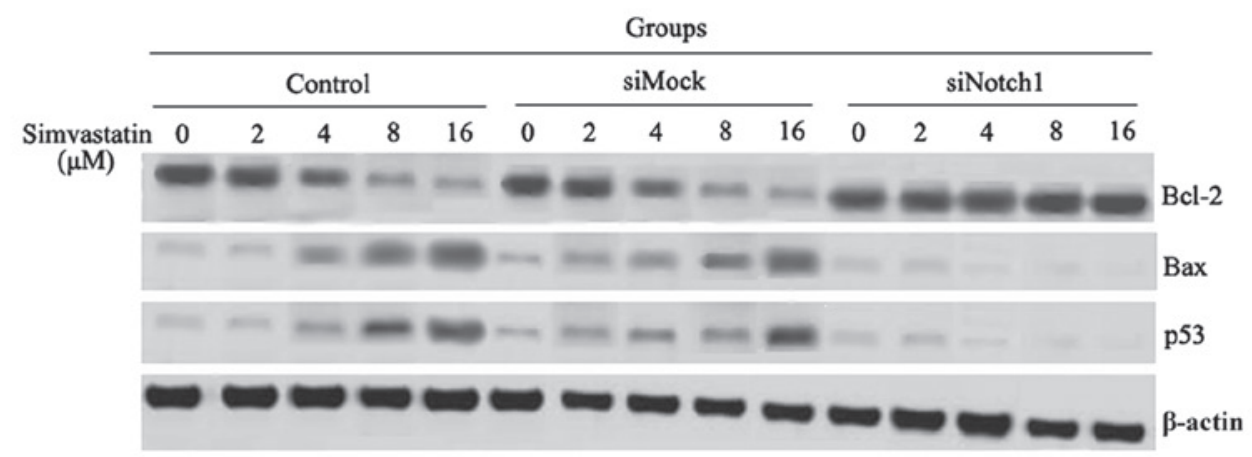

Figure 4. Cell apoptosis induced by simvastatin is weakened and the expression of p53 and Bax decreased when the Notch1 gene was knocked out. (A) Western blot analysis demonstrates the successful knockout of Notch1 in HepG2 cells. (B) Reverse transcription quantitative polymerase chain reaction of Notch1 mRNA expression levels in HepG2 cells following Notch1 knockout. (C) Broken line showing MTT results of proliferation of HepG2 cells following Notch1 knockout. (D) Western blot analysis of protein expression levels of p53, Bcl-2 and Bax in HepG2 cells. Bcl-2, B cell lymphoma 2; Bax, Bcl-2-associated X protein; siNotch1, cells transfected with Notch1 small interfering RNA plasmid; siMock, cells transfected with an empty vector; control, untransfected cells.

A

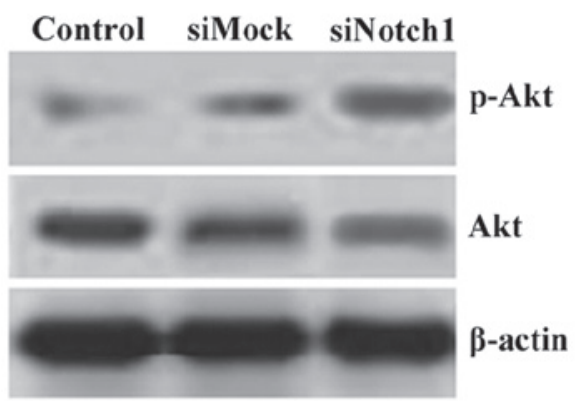

B

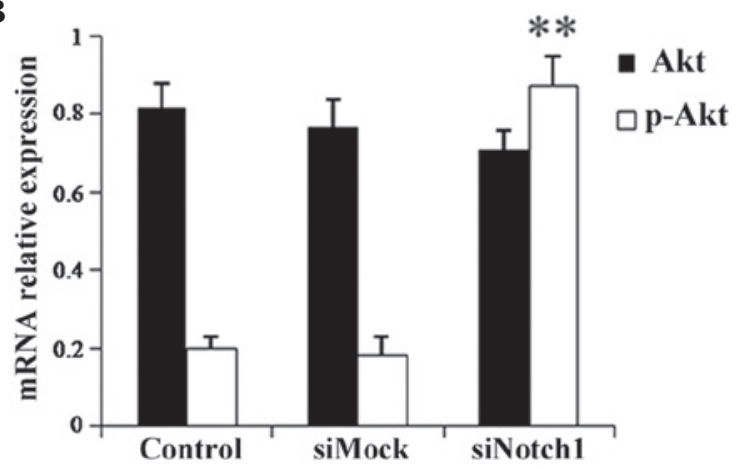

Figure 5. Simvastatin decreases phosphorylation levels of Akt via upregulation of Notch1 expression in HepG2 cells. (A) Western blot analysis was used to determine protein expression levels of Akt and p-Akt in HepG2 cells following Notch1 knockout. (B) Reverse transcription quantitative polymerase chain reaction demonstrated mRNA expression levels of Akt and p-Akt in HepG2 cells following Notch1 knockout. " $\mathrm{P}<0.01$ compared with the control and siMock groups. p-Akt, phosphorylated Akt; siNotch1, cells transfected with Notch1 small interfering RNA plasmid; siMock, cells transfected with an empty vector; control, untransfected cells.

investigate its role in simvastatin-induced apoptosis. As shown in Fig. 4A and B, Notch1 was successfully knocked out in HepG 2 cells. Following Notch1 siRNA transfection, the inhibitory effect of simvastatin on proliferation in HepG2 cells was markedly decreased (Fig. 4C); in addition, mRNA expression levels of p53 and Bax were downregulated compared to those of the control and mock siRNA-transfected groups $(\mathrm{P}<0.05)$ (Fig. 4D).

Simvastatin inhibits the phosphorylation of Akt via upregulation of Notch1 expression in HepG2 cells. Western blot analysis and RT-qPCR were performed in order to investigate the mechanism of simvastatin-induced HepG2 cell apoptosis by detecting the differential expression of the cell survival-associated gene Akt. As shown in Fig. 5, simvastatin inhibited the phosphorylation of Akt in the control and mock siRNA-transfected groups; however, following Notch1 siRNA transfection, the inhibitory effect of simvastatin on p-Akt levels was significantly decreased (Fig. 5). This therefore indicated that simvastatin regulated the phosphorylation of Akt via increasing the expression of Notch1, which, in turn, resulted in HCC cell apoptosis. 


\section{Discussion}

Previous studies have indicated the potential use of statins as cancer therapeutics; one study demonstrated simvastatin was shown to decrease the cellular activity of thioredoxin reductases in HepG2 cells, inhibiting cancer cell proliferation (25). Cancer cells express elevated levels of 3-hydroxy-3-methylglutaryl-coenzyme A reductase and low-density lipid receptor in order to facilitate the increased demand for isoprenoids and lipids (26); this therefore results in the increased sensitivity of cancer cells to statins compared with that of normal cells. However, the mechanisms by which the anti-cancer effects of statins proceed remains to be elucidated. Numerous studies have demonstrated that statins induced apoptosis in cancer cells via various pathways in vitro (27-31); however, there were discrepancy between the results of in vivo and in vitro studies. The biological mechanisms by which statins induce apoptosis and exert anti-proliferative effects in HCC cells are complex. The results of the present study revealed a novel mechanism by which simvastatin exerted cytotoxic effects in the HepG2 and Huh7 cancer cell lines.

Notch1 protein is an essential molecule in the Notch signaling pathway, which has an important role in maintaining the balance between proliferation and differentiation. Previous studies have shown that activated Notch1 inhibited growth in human papillomavirus-positive cervical carcinoma cells and prostate cancer cells, as well as induced cell cycle arrest in small cell lung cancer cells (32-34). In addition, a study in Notch1-deficient mice demonstrated that Notch1 functioned as a tumor suppressor in mouse skin via inhibition of $\beta$-catenin signaling (35). Another previous study provided novel evidence for the potential role of Notch1 signaling in modulating the state of human liver carcinoma $(36,37)$. The results of the present study, showed that simvastatin inhibited HepG2 and Huh7 cell viability and proliferation, as well as promoted apoptosis; in addition, the anti-tumor effect of simvastatin was found to be associated with the Notch1 gene. This therefore indicated that simvastatin may inhibit HCC cell growth via regulation of Notch1 expression. These results were consistent with a previous study which showed that Notch1 arrested the cell cycle of HCC via cyclin A, cyclin D1, cyclin E and cdk4 (38).

The aim of the present study was to investigate the role of Notch1 signaling in simvastatin-induced apoptosis in HepG2 and Huh7 cell by assessing the effect of Notch1 signaling on the expression of the apoptosis-associated proteins p53, Bax, and Bcl-2. The results showed that expression levels of p53 and Bax expression were significantly increased, whereas Bcl-2 expression was significantly decreased in a time- and dose-dependent manner. Previous studies have demonstrated that the overexpression of mutant or wild-type p53 may downregulate $\mathrm{Bcl}-2$ expression, resulting in apoptotic cell death (39). The results of the present study therefore indicated that following cell cycle arrest, Notch1 signaling induced apoptosis via a p53-dependent reduction in Bcl-2 pathway signaling.

Numerous studies have indicated that Akt kinase may be a noval target for cancer therapy (40-43). Experimental models have revealed an association between the regulation of survivin expression and increased Akt activity (44). In prostate cancer cells, survivin expression was shown to be regulated by insulin-like growth factor 1 (IGF-1)-stimulated Akt-mTOR signaling (45), which was impaired following simvastatin treatment (10). Therefore, the present study investigated whether simvastatin-induced apoptosis in HepG2 and Huh7 cells was associated with the Akt pathway. The results demonstrated that simvastatin reduced levels of phosphorylated Akt in HCC cells. However, following Notch1 gene knock out, simvastatin had no effect on p-Akt expression. These results suggested that the mechanism of simvastatin-induced HCC cell apoptosis may proceed via regulation of the expression of the Notch1 gene, which has an important role in the Akt-dependent signaling pathway.

In conclusion, the results of the present study demonstrated that simvastatin treatment induced apoptosis in human HCC cell lines HepG2 and Huh7; in addition, simvastatin decreased cell viability and proliferation in a time- and dose-dependent manner. The anti-tumor effect of simvastatin was found to be associated with p53, Bcl-2 and Bax expression levels, as well as the Akt-dependent signaling pathway. The present study reinforced the anti-tumor effect of statins and provided evidence for the potential beneficial effects of simvastatin in HCC therapy in humans.

\section{Acknowledgements}

The present study was supported by the Science and Technology Plan Projects of Social Development Plans for Public Relations of Shaanxi Province (nos. S2009SF471 and 2012SF2-13).

\section{References}

1. El-Serag HB and Rudolph KL: Hepatocellular carcinoma: epidemiology and molecular carcinogenesis. Gastroenterology 132: 2557-2576, 2007

2. Tsochatzis E, Meyer T, O'Beirne J and Burroughs AK: Transarterial chemoembolisation is not superior to embolisation alone: The recent European Association for the Study of the Liver (EASL)-European Organisation for Research and Treatment of Cancer (EORTC) guidelines. Eur J Cancer 49: 1509-1510, 2013.

3. Cabibbo G, Rolle E, De Giorgio M, et al: Management of cirrhotic patients with hepatocellular carcinoma treated with sorafenib. Expert Rev Anticancer Ther 11: 1807-1816, 2011.

4. Parkin DM: Global cancer statistics in the year 2000. Lancet Onco 2: 533-543, 2001.

5. Jakobisiak M and Golab J: Statins can modulate effectiveness of antitumor therapeutic modalities. Med Res Rev 30: 102-135, 2010.

6. Jakobisiak $M$ and Golab J: Potential antitumor effects of statins (Review). Int J Oncol 23: 1055-1069, 2003.

7. Graaf MR, Richel DJ, van Noorden CJ and Guchelaar HJ: Effects of statins and farnesyltransferase inhibitors on the development and progression of cancer. Cancer Treat Rev 30: 609-641, 2004.

8. Liao JK and Laufs U: Pleiotropic effects of statins. Annu Rev Pharmacol Toxicol 45: 89-118, 2005.

9. Freed-Pastor WA, Mizuno H, Zhao X, et al: Mutant p53 disrupts mammary tissue architecture via the mevalonate pathway. Cell 148: 244-258, 2012.

10. Kochuparambil ST, Al-Husein B, Goc A, Soliman S and Somanath PR: Anticancer efficacy of simvastatin on prostate cancer cells and tumor xenografts is associated with inhibition of Akt and reduced prostate-specific antigen expression. J Pharmacol Exp Ther 336: 496-505, 2011.

11. Wang Y, Xu SL, Wu YZ, et al: Simvastatin induces caspase-dependent apoptosis and activates p53 in OCM-1 cells. Exp Eye Res, 2013.

12. Xu J, Liu X, Chen J, et al: Simvastatin enhances bone marrow stromal cell differentiation into endothelial cells via notch signaling pathway. Am J Physiol Cell Physiol 296: C535-C543, 2009.

13. Zacharek A, Chen J, Cui X, Yang Y and Chopp M: Simvastatin increases notch signaling activity and promotes arteriogenesis after stroke. Stroke 40: 254-260, 2009. 
14. Aster JC, Xu L, Karnell FG, et al: Essential roles for ankyrin repeat and transactivation domains in induction of T-cell leukemia by notch1. Mol Cell Biol 20: 7505-7515, 2000.

15. Bresnick EH, Chu J, Christensen HM, Lin B and Norton J: Linking Notch signaling, chromatin remodeling, and T-cell leukemogenesis. J Cell Biochem Suppl 35 (Suppl.): 46-53, 2000.

16. Wael H, Yoshida R, Kudoh S, et al: Notch signaling controls cell proliferation, apoptosis and differentiation in lung carcinoma. Lung Cancer 85: 131-140, 2014.

17. Ramdass B, Maliekal TT, Lakshmi S, et al: Coexpression of Notch1 and NF- $\kappa$ B signaling pathway components in human cervical cancer progression. Gynecol Oncol 104: 352-361, 2007.

18. Wang Z, Banerjee S, Li Y, et al: Down-regulation of notch-1 inhibits invasion by inactivation of nuclear factor- $\kappa \mathrm{B}$, vascular endothelial growth factor, and matrix metalloproteinase-9 in pancreatic cancer cells. Cancer Res 66: 2778-2784, 2006.

19. Wang J, Fu L, Gu F and Ma Y: Notch1 is involved in migration and invasion of human breast cancer cells. Oncol Rep 26 : 1295-1303, 2011

20. Qi R, An H, Yu Y, et al: Notch1 signaling inhibits growth of human hepatocellular carcinoma through induction of cell cycle arrest and apoptosis. Cancer Res 63: 8323-8329, 2003

21. Yu Y, Zhou XD, Liu YK, et al: Platelets promote the adhesion of human hepatoma cells with a highly metastatic potential to extracellular matrix protein: involvement of platelet P-selectin and GP IIb-IIIa. J Cancer Res Clin Oncol 128: 283-287, 2002.

22. Yu H, Zhao X, Huang S, et al: Blocking Notch1 signaling by RNA interference can induce growth inhibition in HeLa cells. Int J Gynecol Cancer 17: 511-516, 2007.

23. Singh L, Pushker N, Saini N, et al: Expression of pro-apoptotic Bax and anti-apoptotic Bcl-2 proteins in human retinoblastoma. Clin Experiment Ophthalmol: Jul 31, 2014 (Epud ahead of print).

24. Gao J, Yan Q, Liu S and Yang X: Knockdown of EpCAM enhaces the chemosensitivity of breast cancer cells to 5-fluorouracil by downregulating the antiapoptotic factor Bcl-2. PLoS One 9: e102590, 2014

25. Ekström L, Johansson M, Monostory K, et al: Simvastatin inhibits the core promoter of the TXNRDl gene and lowers cellular TrxR activity in HepG2 cells. Biochem Biophys Res Commun 430: 90-94, 2013

26. Liao JK: Isoprenoids as mediators of the biological effects of statins. J Clin Invest 110: 285-288, 2002.

27. Shimada T, Takeshita Y, Murohara T, et al: Angiogenesis and vasculogenesis are impaired in the precocious-aging klotho mouse. Circulation 110: 1148-1155, 2004.

28. Seeger H, Wallwiener D and Mueck A: Statins can inhibit proliferation of human breast cancer cells in vitro. Exp Clin Endocrinol Diabetes 111: 47-48, 2003.
29. Mandal CC, Ghosh-Choudhury N, Yoneda T, Choudhury GG and Ghosh-Choudhury N: Simvastatin prevents skeletal metastasis of breast cancer by an antagonistic interplay between p53 and CD44. J Biol Chem 286: 11314-11327, 2011.

30. Hwang KE, Na KS, Park DS, et al: Apoptotic induction by simvastatin in human lung cancer A549 cells via Akt signaling dependent down-regulation of survivin. Invest New Drugs 29: 945-952, 2011.

31. Hussain B, Saleh GM, Sivaprasad S and Hammond CJ: Changing from Snellen to LogMAR: debate or delay? Clin Experimental Ophthalmol 34: 6-8, 2006.

32. Sriuranpong V, Borges MW, Ravi RK, et al: Notch signaling induces cell cycle arrest in small cell lung cancer cells. Cancer Res 61: 3200-3205, 2001.

33. Shou J, Ross S, Koeppen H, de Sauvage FJ and Gao WQ: Dynamics of notch expression during murine prostate development and tumorigenesis. Cancer Res 61: 7291-7297, 2001.

34. Talora C, Sgroi DC, Crum CP and Dotto GP: Specific down-modulation of Notch1 signaling in cervical cancer cells is required for sustained HPV-E6/E7 expression and late steps of malignant transformation. Genes Dev 16: 2252-2263, 2002.

35. Nicolas M, Wolfer A, Raj K, et al: Notch1 functions as a tumor suppressor in mouse skin. Nature Genet 33: 416-421, 2003.

36. Ahn S, Hyeon J and Park CK: Notch1 and Notch 4 are markers for poor prognosis of hepatocellular carcinoma. Hepatobiliary Pancreat Dis Int 12: 286-294, 2013.

37. Zhou L, Zhang N, Song W, et al: The significance of Notch1 compared with Notch3 in high metastasis and poor overall survival in hepatocellular carcinoma. PLoS One 8: e57482, 2013.

38. Masaki T, Shiratori Y, Rengifo W, et al: Cyclins and cyclin-dependent kinases: Comparative study of hepatocellular carcinoma versus cirrhosis. Hepatology 37: 534-543, 2003.

39. Basu A and Haldar S: The relationship between BcI2, Bax and p53: consequences for cell cycle progression and cell death. Mol Hum Reprod 4: 1099-1109, 1998

40. Somanath P, Vijai J, Kichina J, Byzova T and Kandel E: The role of PAK-1 in activation of MAP kinase cascade and oncogenic transformation by Akt. Oncogene 28: 2365-2369, 2009.

41. Goc A, Al-Husein B, Kochuparambil ST, et al: PI3 kinase integrates Akt and MAP kinase signaling pathways in the regulation of prostate cancer. Int J Oncol 38: 267-277, 2011.

42. Crowell JA, Steele VE and Fay JR: Targeting the AKT protein kinase for cancer chemoprevention. Mol Cancer Ther 6: 2139-2148, 2007.

43. Davies MA: Regulation, role, and targeting of Akt in cancer. J Clin Oncol 29: 4715-4717, 2011.

44. Amaravadi R and Thompson CB: The survival kinases Akt and Pim as potential pharmacological targets. J Clin Invest 115: 2618-2624, 2005

45. Vaira V,Lee CW, Goel HL, et al: Regulation of survivin expression by IGF-1/mTOR signaling. Oncogene 26: 2678-2684, 2006. 